\title{
On quasisymmetric embeddings of the Brownian map and continuum trees
}

\author{
Sascha Troscheit ${ }^{1}[$
}

Received: 16 December 2019 / Revised: 27 November 2020 / Accepted: 23 December 2020 /

Published online: 12 January 2021

(c) The Author(s) 2021

\begin{abstract}
The Brownian map is a model of random geometry on the sphere and as such an important object in probability theory and physics. It has been linked to Liouville Quantum Gravity and much research has been devoted to it. One open question asks for a canonical embedding of the Brownian map into the sphere or other, more abstract, metric spaces. Similarly, Liouville Quantum Gravity has been shown to be "equivalent" to the Brownian map but the exact nature of the correspondence (i.e. embedding) is still unknown. In this article we show that any embedding of the Brownian map or continuum random tree into $\mathbb{R}^{d}, \mathbb{S}^{d}, \mathbb{T}^{d}$, or more generally any doubling metric space, cannot be quasisymmetric. We achieve this with the aid of dimension theory by identifying a metric structure that is invariant under quasisymmetric mappings (such as isometries) and which implies infinite Assouad dimension. We show, using elementary methods, that this structure is almost surely present in the Brownian continuum random tree and the Brownian map. We further show that snowflaking the metric is not sufficient to find an embedding and discuss continuum trees as a tool to studying "fractal functions".
\end{abstract}

Mathematics Subject Classification Primary 60D05; Secondary 28A80 - 05C80 . 37C45

\section{Introduction}

Over the past few years two important models of random geometry of the sphere $\mathbb{S}^{2}$ emerged. One was originally motivated by string theory and conformal field theory in physics and is known as Liouville Quantum Gravity. The other is known as the

ST was initially supported by NSERC Grants 2016-03719 and 2014-03154, and the University of Waterloo.

Sascha Troscheit

sascha.troscheit@univie.ac.at

https://www.mat.univie.ac.at/ troscheit/

1 Faculty of Mathematics, University of Vienna, Oskar Morgenstern Platz 1, 1090 Vienna, Austria 
Brownian map, which originated from the study of large random planar maps. The Brownian map turned out to be a universal limit of random planar maps of $\mathbb{S}^{2}$ and both models have attracted a great deal of interest over the past few years.

We say that a map is planar if it is an embedding of a finite connected graph onto $\mathbb{S}^{2}$ with no edge-crossings. A quadrangulation is a planar map where all its faces are incident to exactly four edges, where edges incident to only one face are counted twice (for both "sides" of the edge). Let $Q_{n}$ be the set of all rooted ${ }^{1}$ quadrangulations with $n$ vertices. This set is finite and we randomly choose a specific quadrangulation $q_{n} \in Q_{n}$ with the uniform probability measure. Let $d_{g r}$ be the graph metric. ${ }^{2}$ It was independently proven by Le Gall [17] and by Miermont [21] that the sequence $\left(q_{n}, n^{-1 / 4} d_{g r}\right)$ converges in distribution to a random limit object $\left(\mathbf{m}_{\infty}, D\right)$, known as the Brownian map, in the space isometry classes of all compact sets $\left(\mathbb{K}, d_{G H}\right)$ equipped with the Gromov-Hausdorff distance. Remarkably, Le Gall also established that this object is universal in the sense that not just quadrangulations, but also uniform triangulations and $2 n$-angulations $(n \geq 2)$ converge in distribution to the same object, up to a rescaling that only depends on the type of $p$-angulation, see [17] and the survey [18]. Notably, the Brownian map is homeomorphic to $\mathbb{S}^{2}$ but has Hausdorff dimension 4 , indicating that the homeomorphism is highly singular. Finding such a canonical mapping is still an active research area and in this article we show, using elementary facts from probability and dimension theory, that the Brownian map has an almost sure metric property that we coin starry. This property implies that the Brownian map and its images under quasisymmetric mappings has infinite Assouad dimension and hence cannot be embedded by quasisymmetric mappings (such as isometries) into finite dimensional manifolds.

The other important model of random surfaces is known as Liouville Quantum Gravity (LQG) which is defined in terms of a real parameter $\gamma$. The LQG is a random geometry based on the Gaussian Free Field (GFF), a random construction that can be considered a higher dimensional variant of Brownian motion. Crucially, the law of the GFF is conformally invariant. When $\gamma=\sqrt{8 / 3}$, the resulting random surface has long been conjectured to be equivalent to the Brownian map. Very recently, work began to unify the two models and although the exact nature of this equivalency (that is, a canonical embedding) is still unknown, major progress was made by Miller and Sheffield in their series of works [23-25]; see also the recent survey [22]. In particular, they showed that the Brownian map and LQG share the same axiomatic properties and that there exists a homeomorphism of the Brownian map onto $\mathbb{S}^{2}$ which realises LQG. As above, the quasisymmetrically invariant starry property implies that no such embedding can be quasisymmetric. In [15], Gwynne, Miller, and Sheffield gave an explicit construction of the embedding as the limit of conformal embeddings, showing that the discretised Brownian disk converges to the conformal embedding of the continuum Brownian disk. The latter corresponds to $\sqrt{8 / 3}-\mathrm{LQG}$.

Before describing our results and background in detail, we remark that the Brownian map can also be obtained from another famous random space: the Brownian

\footnotetext{
1 A graph is said to be rooted if there exists a unique oriented and distinguished edge, called the root.

2 The distance $d_{g r}(v, w)$ between two vertices $v, w$ in a finite connected graph is equal to the length of the shortest path between $v$ and $w$. If $v=w$, we set $d_{g r}=0$.
} 
continuum random tree. The Brownian continuum random tree (CRT) is a continuum tree that was introduced and studied by Aldous in [2-4]. It appears in many seemingly disjoint contexts such as the scaling limit of critical Galton-Watson trees and Brownian excursions using a "least intermediate point" metric. This ubiquity led to the CRT becoming an important object in probability theory in its own right. The Brownian map can be constructed from the CRT by another change in metric and it is this description that allows access to a very different set of probabilistic tools on which our proofs are based. In fact, we first prove that the CRT is starry and thus also cannot be embedded into finite dimensional manifolds almost surely. Note that this is also in stark contrast with the work on conformal weldings of the CRT in [19] and implies much higher singularity of embeddings than previously known.

This article is structured as follows: In Sect. 2, we recall the definition of the Assouad dimension and its use in embedding theory. We will also introduce the starry property for metric spaces in Sect. 2.2 and show that it is invariant under quasisymmetric mappings and implies infinite Assouad dimension. In Sect. 3, we define the Brownian continuum random tree via the Brownian excursion and show that the CRT is starry almost surely. We conclude that it cannot be embedded into $\mathbb{R}^{d}$ for any $d \in \mathbb{N}$ using quasisymmetric mappings. In Sect. 4, we define the Brownian map through the CRT and prove that the Brownian map is starry, also. In particular, Theorem 2.4 states that quasisymmetric images of starry metric spaces have infinite Assouad dimension (and are thus not doubling), Theorem 3.5 proves that the CRT is starry almost surely, and Theorem 4.1 shows that the Brownian map is starry. Section 5 finishes this article by containing a discussion of our results from a fractal geometric point of view. Throughout, we postpone proofs until the end of their respective section.

\section{Assouad dimension and embeddings}

The Assouad dimension is an important tool in the study of embedding problems. It was first introduced by Patrice Assouad in [5]. More recently, the exact determination of the Assouad dimension for random and deterministic subsets of Euclidean space has revealed intricate relations with separation properties in the study of fractal sets. Notably, the Assouad dimension of random sets tends to be "as big as possible", see e.g. [12], and that for self-conformal subsets of $\mathbb{R}$, Ahlfors regularity is equivalent to the Assouad dimension coinciding with the Hausdorff dimension, see [1].

\subsection{Assouad dimension}

Formally, let $(X, d)$ be a metric space and write $N(X, r)$ for the minimal number of sets of diameter at most $r$ needed to cover $X$. We set $N(X, r)=\infty$ if no such collection exists.

Let $B(x, r)$ be the closed ball in $X$ of radius $r>0$. The Assouad dimension is then given by 


$$
\operatorname{dim}_{\mathrm{A}}(X)=\inf \left\{\alpha:(\exists C>0)(\forall 0<r<R<1) \sup _{x \in X} N(B(x, R), r) \leq C\left(\frac{R}{r}\right)^{\alpha}\right\} .
$$

There are several important generalisations and variations of the Assouad dimension such as the quasi-Assouad dimension and the Assouad spectrum. The latter fixes the relationship between $r$ and $R$ by a parameter $\theta$, i.e. $r=R^{1 / \theta}$, whereas the former is a slightly more regularised version of the Assouad dimension. One could certainly ask the questions that arise here of these variants and we forward the interested reader to the survey [11] for an overview.

The Assouad dimension is always an upper bound to the Hausdorff dimension but coincides in many "natural" examples such as $k$-dimensional Riemannian manifolds. However, they can also differ widely in general metric spaces and it is possible to construct a space $X$ such that $X$ is countable with Hausdorff dimension 0 but has infinite Assouad dimension; see e.g. Proposition 2.7. These sets are however somewhat pathological and it would be interesting to find 'natural' metric spaces that have low Hausdorff and box-counting dimension but are 'big' in the sense of Assouad dimension. A natural candidate are random sets since they tend to have a regular average behaviour, giving low Hausdorff dimension, but have rare but very 'thick' regions; see $[12,13,27,28]$. These regions are detected by the Assouad dimension and, as we will show in this article, are sufficient to give infinite Assouad dimension for the Brownian map and CRT.

The Assouad dimension is strongly related to the metric notion of doubling: a metric space has finite Assouad dimension if and only if it is doubling. Further, the Assouad dimension is invariant under bi-Lipschitz mappings and is a useful indicator when a space is or is not embeddable. Because of this invariance, a metric space $(X, d)$ with Assouad dimension $s_{a}=\operatorname{dim}_{\mathrm{A}} X$ cannot be embedded into $\mathbb{R}^{\left\lceil s_{a}\right\rceil-1}$ with bi-Lipschitz mappings. The converse is not quite true, but "snowflaking" the metric by some $\alpha>0$ allows a bi-Lipschitz embedding [5].

Theorem 2.1 (Assouad Embedding Theorem) Let $(X, d)$ be a metric space with finite Assouad dimension. Then there exists $C>1, N \in \mathbb{N}, 1 / 2<\alpha<1$, and an injection $\phi: X \rightarrow \mathbb{R}^{N}$ such that

$$
C^{-1} d(x, y)^{\alpha} \leq|\phi(x)-\phi(y)| \leq C d(x, y)^{\alpha} \quad \forall x, y \in X
$$

Explicit bounds on $N$ and $\alpha$ can be obtained from the Assouad dimension, see e.g. [8].

\subsection{Starry metric spaces}

While the Assouad dimension is invariant under bi-Lipschitz mappings, this is not true for the more general notion of quasisymmetric mappings. We recall the definition of a quasisymmetric mapping. 
Definition 2.2 Let $\left(X, d_{X}\right)$ and $\left(Y, d_{Y}\right)$ be metric spaces. A homeomorphism $\phi: X \rightarrow Y$ is called $\Psi$-quasisymmetric if there exists an increasing function $\Psi:(0, \infty) \rightarrow(0, \infty)$ such that for any three distinct points $x, y, z \in X$,

$$
\frac{d_{Y}(\phi(x), \phi(y))}{d_{Y}(\phi(x), \phi(z))} \leq \Psi\left(\frac{d_{X}(x, y)}{d_{X}(x, z)}\right) .
$$

A basic example of quasi-symmetric mappings are isometric embeddings but the notion of quasisymmetric mappings generalises it by allowing a uniformly controlled distortion. In Euclidean space quasisymmetric mappings correspond to quasi-conformal mappings. That is, if $\phi: \Omega \rightarrow \Omega^{\prime}$, where $\Omega, \Omega^{\prime} \subset \mathbb{R}^{d}$ are open and $\phi$ is $\Psi$-quasisymmetric, then $\phi$ is also $K$-quasiconformal for some $K$ depending only on the function $\Psi$. A similar statement holds in the other direction.

In [20], Mackay and Tyson study the Assouad dimension under symmetric mappings. In particular, it is possible to lower the Assouad dimension by quasisymmetric mappings (see also [29]) and they introduce the notion of the conformal Assouad dimension. The conformal Assouad dimension of a metric space $(X, d)$ is defined as the infimum of the Assouad dimension of all quasisymmetric images of $X$. This notion has been subsequently explored for many examples of deterministic sets, such as self-affine carpets in [16].

Here we define the structure of an approximate $n$-star, which, heuristically, is the property that a space contains $n$ distinct points that are roughly equidistant to a central point with every geodesic between them going near the centre, the extent of which is controlled by the parameters $A$ and $\mu$.

Definition 2.3 A metric space $(X, d)$ is said to contain an $(A, \eta)$-approximate $n$-star if there exists $A>1$ and $0<\eta<1, \varrho>0$, such that $A-\eta>1+\eta \Leftrightarrow \eta<(A-1) / 2$ as well as $\varrho>0$ and a set of points $\left\{x_{0}, \ldots, x_{n}\right\} \subseteq(X, d)$ satisfying

$$
(A-\eta) \varrho \leq d\left(x_{i}, x_{j}\right) \leq A \varrho \text { for all } i, j \in\{1, \ldots, n\} \text { with } i \neq j
$$

and

$$
\varrho \leq d\left(x_{0}, x_{i}\right) \leq(1+\eta) \varrho \text { for all } i \in\{1, \ldots, n\}
$$

We say that a metric space $(X, d)$ is starry if there exist uniform $A>1$ and $0<\eta<$ $(A-1) / 2$ such that $(X, d)$ contains an $\left(A_{n}, \eta\right)$-approximate $n$-star for all $n$, where $A \leq A_{n}$.

We note that any $(A, \eta)$-approximate $n$-star is also an $(A, \zeta)$-approximate $m$-star for all $m \leq n$ and $\zeta \geq \eta$, provided that $1+\zeta<A-\zeta$. Further, $A$ is always bounded above by the triangle inequality, since $(A-\eta) \varrho \leq d\left(x_{1}, x_{2}\right) \leq d\left(x_{1}, x_{0}\right)+d\left(x_{0}, x_{2}\right) \leq$ $2(1+\eta) \varrho$, giving $1<1+2 \eta<A \leq 2+3 \eta<5$.

Our main theorem in this section states that all quasisymmetric images of starry metric spaces (including the identity) have infinite Assouad dimension. Essentially, being starry means that the conformal Assouad dimension is maximal. 
Theorem 2.4 Let $(X, d)$ be a starry metric space and let $\phi$ be a quasisymmetric mapping $\phi:(X, d) \rightarrow\left(\phi(X), d_{\phi}\right)$. Then $\operatorname{dim}_{\mathrm{A}} \phi(X)=\infty$.

Recall that a metric space $(X, d)$ is said to be doubling if there exists a constant $K>0$ such that the ball $B(x, r) \subseteq X$ can be covered by at most $K$ balls of radius $r / 2$ for all $x \in X$ and $r>0$. Given that the Assouad dimension of a metric space is finite if and only if it is doubling, see e.g. [10, Theorem 13.1.1], we obtain

Corollary 2.5 Let $\left(X, d_{X}\right)$ be a starry metric space and $\left(Y, d_{Y}\right)$ be a doubling metric space. Then $X$ is not doubling and any embedding $\phi: X \rightarrow Y$ cannot be quasisymmetric.

It is a simple exercise to see that any $s$-Ahlfors regular space ${ }^{3}$ has Assouad (and Hausdorff) dimension equal to $s$ and so $\mathbb{R}^{d}$ has Assouad dimension $d$. Similarly, any $d$-dimensional Riemannian space is $d$-Ahlfors regular, as it supports a $d$-regular volume measure. Our result immediately implies that any starry metric space cannot be embedded into such finite dimensional spaces by quasisymmetric (and hence biLipschitz) mappings.

Careful observation of the estimates in the proof of Theorem 2.4 shows that snowflaking does not allow a quasisymmetric or bi-Lipschitz embedding. That is, there is no analogy of the Assouad embedding theorem (Theorem 2.1) for starry metric spaces and we get the stronger statement.

Corollary 2.6 Let $\left(X, d_{X}\right)$ be a starry metric space and let $\left(Y, d_{Y}\right)$ be a doubling metric space (such as an $s$-Ahlfors regular space with $s \in[0, \infty)$ ). Let $\alpha \in(0,1]$, $\Psi:(0, \infty) \rightarrow(0, \infty)$ be an increasing function, and $\phi: X \rightarrow Y$ be an embedding of $X$ into $Y$. Then $\phi$ cannot satisfy

$$
\frac{d_{Y}(\phi(x), \phi(y))}{d_{Y}(\phi(x), \phi(z))} \leq \Psi\left(\frac{d_{X}(x, y)^{\alpha}}{d_{X}(x, z)^{\alpha}}\right)
$$

for distinct $x, y, z \in X$.

In analogy to the observation that every set $X \subset \mathbb{R}^{d}$ with Assouad dimension $d \in \mathbb{N}$ must contain $[0,1]^{d}$ as a weak tangent, see [14], one might think that a metric space with infinite Assouad dimension must also be starry. However, that is not true.

Proposition 2.7 There exists a countable and bounded metric space with infinite Assouad dimension that is not starry.

We will give an example in the next section.

\subsection{Proofs for Sect. 2}

Proof of Theorem 2.4 We argue by contradiction. Assume $\operatorname{dim}_{\mathrm{A}} \phi(X)<\infty$. Then there exists $s>0$ and $C>1$ such that $N\left(B_{d_{\phi}}(x, R), r\right) \leq C \cdot(R / r)^{s}$ for all

\footnotetext{
${ }^{3}$ A metric space $X$ is $s$-Ahlfors regular if it supports a Radon measure $\mu$ such that $\mu(B(x, r)) \sim r^{s}$ for all $x \in X$ and $0<r<\operatorname{diam} X$.
} 
$0<r<R<\operatorname{diam}_{d_{\phi}} \phi(X)$ and $x \in \phi(X)$. We assume that $X$ is starry and thus there exist $A$ and $\eta$ such that $X$ has $(A, \eta)$-approximate $n_{k}$-stars. Pick $k$ such that $n_{k}>C \cdot(4 \Psi(1) \Psi(1+\eta))^{s}$, where $\Psi:(0, \infty) \rightarrow[0, \infty)$ is the scale distortion function of the quasisymmetric mapping $\phi$.

Let $x_{i}, \varrho$, be the points and size of the approximate $n_{k}$-star. First note that distances relative to the centre $x_{0}$ are preserved. That is, for all $i \in\left\{1, \ldots, n_{k}\right\}$,

$$
\frac{d_{\phi}\left(\phi\left(x_{0}\right), \phi\left(x_{i}\right)\right)}{d_{\phi}\left(\phi\left(x_{0}\right), \phi\left(x_{j}\right)\right)} \leq \Psi\left(\frac{d\left(x_{0}, x_{i}\right)}{d\left(x_{0}, x_{j}\right)}\right) \leq \Psi\left(\frac{(1+\eta) \varrho}{\varrho}\right) \leq \Psi(1+\eta) .
$$

One deduces a lower bound by taking the inverse

$$
\frac{1}{\Psi(1+\eta)} \leq \frac{d_{\phi}\left(\phi\left(x_{0}\right), \phi\left(x_{i}\right)\right)}{d_{\phi}\left(\phi\left(x_{0}\right), \phi\left(x_{j}\right)\right)} \leq \Psi(1+\eta)
$$

for all $i, j \in\left\{1, \ldots, n_{k}\right\}$. Note that $B_{d_{\phi}}\left(\phi\left(x_{0}\right), R\right)=\left\{y \in \phi(X): d_{\phi}\left(\phi\left(x_{0}\right), y\right) \leq\right.$ $R\}$ contains $\left\{\phi\left(x_{0}\right), \ldots, \phi\left(x_{n}\right)\right\}$ for $R=\Psi(1+\eta) \min _{k} d_{\phi}\left(\phi\left(x_{0}\right), \phi\left(x_{k}\right)\right)$.

Let $i \neq j \in\left\{1, \ldots, n_{k}\right\}$. We estimate

$$
\frac{d_{\phi}\left(\phi\left(x_{0}\right), \phi\left(x_{i}\right)\right)}{d_{\phi}\left(\phi\left(x_{i}\right), \phi\left(x_{j}\right)\right)} \leq \Psi\left(\frac{d\left(x_{0}, x_{i}\right)}{d\left(x_{i}, x_{j}\right)}\right) \leq \Psi\left(\frac{1+\eta}{A-\eta}\right) \leq \Psi(1) .
$$

and so obtain $d_{\phi}\left(\phi\left(x_{i}\right), \phi\left(x_{j}\right)\right) \geq d_{\phi}\left(\phi\left(x_{0}\right), \phi\left(x_{i}\right)\right) / \Psi(1) \geq(1 / \Psi(1)) \min _{k} d_{\phi}\left(\phi\left(x_{0}\right)\right.$, $\left.\phi\left(x_{k}\right)\right)$. Set $r=(1 /(4 \Psi(1))) \min _{k} d_{\phi}\left(\phi\left(x_{0}\right), \phi\left(x_{k}\right)\right)$ and let $y_{i}, y_{j} \in \phi(X)$ be such that $\phi\left(x_{i}\right) \in B_{d_{\phi}}\left(y_{i}, r\right)$ and $\phi\left(x_{j}\right) \in B_{d_{\phi}}\left(y_{j}, r\right)$ for $i \neq j \in\left\{1, \ldots, n_{k}\right\}$. Then,

$$
\begin{aligned}
& B_{d_{\phi}}\left(y_{i}, r\right) \cap B_{d_{\phi}}\left(y_{j}, r\right) \\
& \quad=\left\{z \in \phi(X): d_{\phi}\left(z, y_{i}\right)<r \text { and } d_{\phi}\left(z, y_{j}\right)<r\right\} \\
& \quad \subseteq\left\{z \in \phi(X): d_{\phi}\left(z, \phi\left(x_{i}\right)\right)<2 r \text { and } d_{\phi}\left(z, \phi\left(x_{j}\right)\right)<2 r\right\} \\
& \quad \subseteq\left\{z \in \phi(X): d_{\phi}\left(\phi\left(x_{i}\right), z\right)+d_{\phi}\left(z, \phi\left(x_{j}\right)\right)\right. \\
& \left.\quad<4 r=(1 / \Psi(1)) \min _{k} d_{\phi}\left(\phi\left(x_{0}\right), \phi\left(x_{i}\right)\right)\right\} \\
& \quad=\varnothing
\end{aligned}
$$

by the triangle inequality and our estimate ${ }^{4}$ for $d_{\phi}\left(\phi\left(x_{i}\right), \phi\left(x_{j}\right)\right)$. We conclude that any $r$-cover of $\left\{\phi\left(x_{1}\right), \ldots, \phi\left(x_{n_{k}}\right)\right\}$ must consist of at least $n_{k}$ balls as no single $r$-ball can cover two distinct points. Hence,

$$
N\left(B_{d_{\phi}}\left(\phi\left(x_{0}\right), R\right), r\right) \geq n_{k} \quad \text { and } \quad \frac{R}{r}=4 \Psi(1) \Psi(1+\eta) .
$$

But then

$C(4 \Psi(1) \Psi(1+\eta))^{s}<n_{k} \leq N\left(B_{d_{\phi}}\left(\phi\left(x_{0}\right), R\right), r\right) \leq C\left(\frac{R}{r}\right)^{s}=C(4 \Psi(1+\eta) \Psi(1))^{s}$,

\footnotetext{
4 This also shows that the starry property is invariant under quasisymmetric mappings.
} 
a contradiction.

Proof of Proposition 2.7 We construct an explicit example of a metric space that is bounded, has infinite Assouad dimension, but is not starry. Consider the set of points $(n, m) \in \mathbb{N} \times \mathbb{N}$ with the pseudometric

$$
d\left((n, m),\left(n^{\prime}, m^{\prime}\right)\right)= \begin{cases}0 & \text { if } n=n^{\prime} \text { and } m=m^{\prime} \\ 0 & \text { if } n=n^{\prime}, \min \left\{m, m^{\prime}\right\}=1 \text { and } \max \left\{m, m^{\prime}\right\}>n \\ 0 & \text { if } n=n^{\prime} \text { and } \min \left\{m, m^{\prime}\right\}>n \\ 2^{-n} & \text { if } n=n^{\prime} \text { and none of the above } \\ 2 \sum_{k=\min \left\{n, n^{\prime}\right\}}^{\max \left\{n, n^{\prime}\right\}} k^{-2} & \text { if } n \neq n^{\prime} .\end{cases}
$$

Since $d((n, m),(n, m))=0$ and the distance function is symmetric, we only need to check the triangle inequality. Let $(n, m),\left(n^{\prime}, m^{\prime}\right),\left(n^{\prime \prime}, m^{\prime \prime}\right)$ be points in our space. If $n \neq n^{\prime \prime}$, then

$$
\begin{aligned}
& d\left((n, m),\left(n^{\prime \prime}, m^{\prime \prime}\right)\right)=2 \sum_{k=\min \left\{n, n^{\prime \prime}\right\}}^{\max \left\{n, n^{\prime \prime}\right\}} k^{-2} \leq 2 \sum_{k=\min \left\{n, n^{\prime}, n^{\prime \prime}\right\}}^{\max \left\{n, n^{\prime}, n^{\prime \prime}\right\}} k^{-2} \\
& \leq 2 \sum_{k=\min \left\{n, n^{\prime}\right\}}^{\max \left\{n^{\prime}, n^{\prime \prime}\right\}} k^{-2}+2 \sum_{k=\min \left\{n^{\prime}, n^{\prime \prime}\right\}}^{\max \left\{n, n^{\prime}\right\}} k^{-2}=d\left((n, m),\left(n^{\prime}, m^{\prime}\right)\right)+d\left(\left(n^{\prime}, m^{\prime}\right),\left(n^{\prime \prime}, m^{\prime \prime}\right)\right) .
\end{aligned}
$$

If, however $n=n^{\prime \prime}$, we may assume that $m \neq m^{\prime \prime}$ and $\min \left\{m, m^{\prime \prime}\right\} \leq n$ and $\left(\min \left\{m, m^{\prime \prime}\right\} \neq 1\right.$ or $\left.\max \left\{m, m^{\prime \prime}\right\} \leq n\right)$ as otherwise the triangle inequality is trivially satisfied. If $n^{\prime} \neq n$, then

$$
d\left((n, m),\left(n^{\prime \prime}, m^{\prime \prime}\right)\right)=2^{-n} \leq 2 n^{-2} \leq 2 \sum_{k=\min \left\{n, n^{\prime}\right\}}^{\max \left\{n, n^{\prime}\right\}} k^{-2} \leq d\left((n, m),\left(n^{\prime}, m^{\prime}\right)\right) .
$$

When $n^{\prime}=n$ then $m^{\prime} \neq m$ or $m^{\prime} \neq m^{\prime \prime}$. Therefore, at least one of $d\left((n, m),\left(n^{\prime}, m^{\prime}\right)\right)$ and $d\left(\left(n^{\prime}, m^{\prime}\right),\left(n^{\prime \prime}, m^{\prime \prime}\right)\right)$ is equal to $2^{-n}$. Again we obtain

$$
d\left((n, m),\left(n^{\prime \prime}, m^{\prime \prime}\right)\right)=2^{-n} \leq d\left((n, m),\left(n^{\prime}, m^{\prime}\right)\right)+d\left(\left(n^{\prime}, m^{\prime}\right),\left(n^{\prime \prime}, m^{\prime \prime}\right)\right)
$$

and $d$ is a pseudometric. In fact, identifying points with distance zero gives the metric space $((\mathbb{N} \times \mathbb{N}) / \approx, d)$ where all $(n, m)$ get identified with $(n, 1)$ for $m>n$.

To see that this space has infinite Assouad dimension one can consider balls $B_{n}$ centered at $(n, 1)$ with diameter $R=2^{-n}$. This ball contains exactly $n$ distinct points $(n, 1),(n, 2), \ldots,(n, n)$ each at distance $R$ from each other. Letting $r=R / 2$ we need $n$ balls of diameter $r$ to cover $B_{n}$. However, there are no uniform constants $C, s$ such that $n \leq C(2)^{s}$ and hence the Assouad dimension is infinite.

To show that this metric space is not starry, we assume for a contradiction that it is and that there exist $1<A, \eta<(A-1) / 2$ and subsets $S_{n}$ that form $\left(A_{n}, \eta\right)$ approximate $n$-stars for $A<A_{n}$. Consider $S_{n}$. It must contain a centre $x_{0}=\left(p_{0}, q_{0}\right) \in$ 
$S_{n}$ and $n$ distinct points in the annulus $D_{n}=B\left(x_{0},(1+\eta) \rho_{n}\right) \backslash B\left(x_{0}, \rho_{n}\right)$. We see that $(1+\eta) \rho_{n}>2^{-n}$ since otherwise $S_{n} \subseteq B_{n}$ and all points in $B_{n}$ are equidistant and do not have a centre. We split the annulus $D_{n}$ in two parts,

$$
D_{n}^{-}=\left\{(p, q) \in \mathbb{N}: p<p_{0} \text { and } \rho_{n} \leq \sum_{k=p}^{p_{0}} k^{-2} \leq(1+\eta) \rho_{n}\right\}
$$

and

$$
D_{n}^{+}=\left\{(p, q) \in \mathbb{N}: p>p_{0} \text { and } \rho_{n} \leq \sum_{k=p_{0}}^{p} k^{-2} \leq(1+\eta) \rho_{n}\right\}
$$

Let $(p, q),\left(p^{\prime}, q^{\prime}\right) \in D_{n}^{+}$be distinct. Then,

$d\left((p, q),\left(p^{\prime}, q^{\prime}\right)\right) \leq \max \left\{2^{-(n+1)}, \sum_{k=p_{0}}^{p} k^{-2}\right\} \leq(1+\eta) \rho_{n}<(A-\eta) \rho_{n} \leq\left(A_{n}-\eta\right) \rho_{n}$.

Thus, $S_{n}$ may contain at most one element in $D_{n}^{+}$and $D_{n}^{-}$contains at least $n-1$ elements. Consider the distinct elements $(p, q),\left(p^{\prime}, q^{\prime}\right) \in D_{n}^{-}$. If $p \neq p^{\prime}$ we must have $d\left((p, q),\left(p^{\prime}, q^{\prime}\right)\right) \leq \sum_{k=p_{0}}^{p} k^{-2} \leq(1+\eta) \rho_{n}<\left(A_{n}-\eta\right) \rho_{n}$. This implies that at least $n-1$ elements in $D_{n}^{-}$are contained a single $B_{p} \subset D_{n}^{-}$. Considering distinct elements $(p, q),\left(p^{\prime}, q^{\prime}\right) \in D_{n}^{-}$with $p=p^{\prime}$, we obtain $d\left((p, q),\left(p^{\prime}, q^{\prime}\right)\right)=2^{-p}$, where $p$ satisfies $\sum_{k=p}^{p_{0}} k^{-2} \leq(1+\eta) \rho_{n}$. Since these points form an approximate $n$-star, we further have $2^{-p}>\left(A_{n}-\eta\right) \rho_{n}>(1+\eta) \rho_{n}$ and so

$$
p^{-2} \leq \sum_{k=p}^{p_{0}} k^{-2} \leq(1+\eta) \rho_{n}<2^{-p}
$$

This implies $p \leq 4$ and so $\# S_{n} \leq 5$, a contradiction as $n$ was arbitrary.

Lastly, the space is bounded as the entire set is contained in $B\left((1,1), 2 \pi^{2} / 6\right)$.

\section{Result for Brownian continuum random trees}

In this section we introduce the concept of $\mathbb{R}$-trees. We define a pseudometric on $[0,1]$ in terms of an excursion function $f$. This pseudometric gives rise to an $\mathbb{R}$-tree which we call the continuum tree of function $f$. Letting $f$ be a generic realisation of the Brownian excursion, we obtain the Brownian continuum random tree (CRT). We will show that the CRT is a starry metric space and thus has infinite Assouad dimension. In Sect. 5 we ask whether this is true for a larger class of functions. 


\section{1 $\mathbb{R}$-trees and excursion functions}

An $\mathbb{R}$-tree is a continuum variant of a tree. It is a metric space that satisfies the following properties.

Definition 3.1 A metric space $(X, d)$ is an $\mathbb{R}$-tree if, for every $x, y \in X$,

(1) there exists a unique isometric mapping $f_{(x, y)}:[0, d(x, y)] \rightarrow X$ such that $f_{(x, y)}(0)=x$ and $f_{(x, y)}(d(x, y))=y$,

(2) if $f:[0,1] \rightarrow X$ is injective with $f(0)=x$ and $f(1)=y$, then

$$
f([0,1])=f_{(x, y)}([0, d(x, y)]) .
$$

We further say that $(X, d)$ is rooted if there is a distinguished point $x \in X$, which we call the root.

Heuristically, $X$ is a connected, but potentially uncountable, union of line segments. Every two points $x, y \in X$ are connected by a unique arc (or geodesic) that is isomorphic to a line segment. We call any $x \in X$ a leaf if $X \backslash\{x\}$ is still connected. In Sect. 4 we will further introduce a labelling on the trees that is used to identify (or glue) certain leaves. The resulting space will be the Brownian map.

There are several canonical ways of generating $\mathbb{R}$-trees, such as the "stick-breaking model" but here we will focus on continuum trees generated by excursion functions.

Definition 3.2 Let $f:[0,1] \rightarrow[0, \infty)$ be a continuous function. We say that $f$ is a (length 1) excursion function if $f(0)=f(1)=0$ and $f(t)>0$ for all $t \in(0,1)$.

These excursion functions are now used to define a new metric on $[0,1]$ that gives a new metric space called the continuum tree.

Definition 3.3 Let $f$ be an excursion function. We call $T_{f}=\left([0,1] / \approx, d_{f}\right)$ the continuum tree with excursion $f$, where $d_{f}$ is the (pseudo-)metric given by

$$
d_{f}(x, y)=f(x)+f(y)-2 \min \{f(t): \min \{x, y\} \leq t \leq \max \{x, y\}\},
$$

and $\approx_{f}$, the equivalence relation on [0,1] defined by $x \approx_{f} y$ if and only if $d_{f}(x, y)=$ 0 .

The resulting metric space is of cardinality the continuum and can be considered a tree with root vertex $0 \approx_{f} 1$, where the lowest common ancestor of the equivalence classes of $x<y$ is given by the equivalence classes of any $t_{0} \in[x, y]$ such that $f\left(t_{0}\right)=\min \{f(t): x \leq t \leq y\}$. We note that while the value for which the minimum is achieved might not be unique in $[0,1]$ with the Euclidean metric, all such points are identified in the metric space $T_{f}=\left([0,1] / \approx_{f}, d_{f}\right)$, where $d_{f}$ is a bona fide metric.

\subsection{The Brownian continuum random tree}

The Brownian continuum random tree was first studied in the comprehensive work of Aldous [2-4]. It is a random metric space that can be obtained with the continuum tree 
metric described above by choosing the normalised Brownian excursion $\mathbf{e}(t)$ as the excursion function. The Brownian excursion can be defined in terms of a Brownian bridge $B(t)$ with parameter $T$, which is a Wiener process conditioned on $B(0)=$ $B(1)=0$. Further, it is well known that the Brownian bridge (with $T=1$ ) can be expressed as $B(t)=W(t)-t W(1)$ where $B(t)$ is independent of $W(1)$. The graph $\{(t, W(t)): t \in[0,1]\}$ and hence $\{(t, B(t)): t \in[0,1]\}$ are compact almost surely and so $B\left(t_{\min }\right)=\min \{B(t): 0 \leq t \leq 1\}$ exists and $t_{\min }$ is almost surely unique. Cutting the Brownian bridge at the minimum and translating, one obtains a Brownian excursion

$$
\mathbf{e}(t)= \begin{cases}B\left(t+t_{\min }\right)-B\left(t_{\min }\right) & 0 \leq t \leq 1-t_{\min } \\ B\left(t-1+t_{\min }\right)-B\left(t_{\min }\right) & 1-t_{\min }<t \leq 1\end{cases}
$$

We use this definition of the Brownian bridge and excursion, as we will need to show a decay of correlations between $\mathbf{e}(s)$ and $\mathbf{e}(t)$, where $s$ and $t$ are in disjoint subintervals of $[0,1]$.

Definition 3.4 Let $\mathbf{e}$ be a Brownian excursion. The random metric space $\left(T_{\mathbf{e}}, d_{\mathbf{e}}\right)=$ $\left([0,1] / \approx_{\mathbf{e}}, d_{\mathbf{e}}\right)$ is called the Brownian continuum random tree (CRT).

The CRT also appears as the limit object of the stick-breaking model and rescaled critical Galton-Watson trees as the number of nodes is taken to infinity. As such, the CRT is an important object in probability theory. It also appears in the construction of the Brownian map, which we will recall in Sect. 4.

Our main result in this section is that the CRT is starry. However, we establish a slightly stronger result below, which we will need in the proof that the Brownian map is starry.

Theorem 3.5 Let $\mathbf{e}$ be a Brownian excursion and $T_{\mathbf{e}}$ be the associated Brownian continuum random tree. Then, for every $n, T_{\mathbf{e}}$ contains infinitely many approximate $n$-stars, almost surely. In particular, $T_{\mathbf{e}}$ is almost surely starry.

Therefore, by Theorem 2.4 and Corollary 2.5, the Brownian excursion has infinite Assouad dimension and cannot be embedded into finite dimensional manifolds using quasisymmetric mappings.

We end this section by noting that, from a fractal geometry standpoint, the continuum tree metric could hold the key to a better understanding of "fractal functions" such as the Weierstraß functions and general self-affine functions, see Sect. 5.

\subsection{Proofs of Sect. 3}

To prove that $T_{\mathbf{e}}$, and later that the Brownian map, is starry, we partition [1/2, 3/4) into countably many disjoint intervals $A_{n}^{m}$ and show that the processes on these intervals are "almost" independent. Let $A_{n}^{k}=\left[a(n, k), a(n, k)+2^{-(n+k+2)}\right]$, where $a(n, k)=$ $\frac{3}{4}-2^{-(n+k+1)}\left(2^{k-1}+1\right)$. Noting that $a(n, k+1)-a(n, k)=2^{-(n+k+2)}$ and $a(n+$ $1,1)>a(n, k)$ for all $n, k \in \mathbb{N}$ we see that the interiors of $A_{n}^{k}$ and $A_{n^{\prime}}^{k^{\prime}}$ are disjoint whenever $n \neq n^{\prime}$ or $k^{\prime} \neq k$. Further, $\bigcup_{n, k \in \mathbb{N}} A_{n}^{k}=\left[\frac{1}{2}, \frac{3}{4}\right)$ and the half-open intervals 
[inf $A_{n}^{k}, \sup A_{n}^{k}$ ) form a countable partition of $\left[\frac{1}{2}, \frac{3}{4}\right)$. We require the following wellknown result that allows us to estimate a Wiener process with a given function. In particular, this follows directly from the construction of the classical Wiener measure on the classical Wiener space and the fact that this measure is strictly positive, see e.g. $[26, \S 8]$.

Lemma 3.6 Let $W(t)$ be a Wiener process on [0, 1]. Then, for every continuous function $f \in C[0,1]$ and $\varepsilon>0$,

$$
\mathbb{P}\left(\|W(t)+f(0)-f(t)\|_{\infty}<\varepsilon\right)>0 .
$$

We will use this lemma with a "zig-zag" function (see Fig. 1) that will give the correct structure on $[0,1]$ when applied with the continuum tree metric. Let $F_{n}(x):[0,1] \rightarrow$ $\mathbb{R}_{\geq 0}$, where

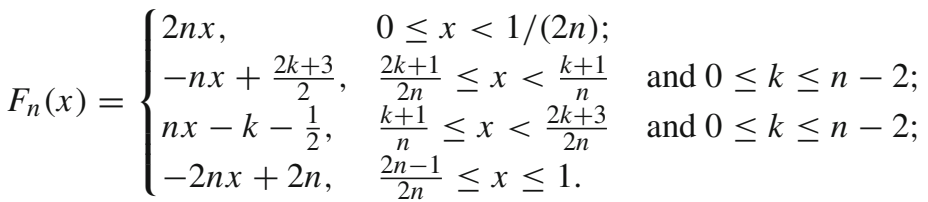

The function $F_{n}$ is an excursion function $\left(F_{n}(0)=0=F_{n}(1)\right)$ that is continuous and linear between the local maxima $F_{n}((k+1) / n)=1$ and local minima $F_{n}((2 k+1) /(2 n))=1 / 2$ in $(0,1)$. We next show that the Brownian excursion contains arbitrarily good approximations to this function for all $n$.

Lemma 3.7 Let $\mathbf{e}(s)$ be a Brownian excursion. Then, almost surely, for every $n$ large enough, there exists infinitely many pairwise disjoint non-trivial intervals $\left[s_{n}^{k}, t_{n}^{k}\right] \subset$ $[0,1], k \in \mathbb{N}$, such that

$$
\sup _{s \in\left[s_{n}^{k}, t_{n}^{k}\right]}\left|\mathbf{e}(s)-\mathbf{e}\left(s_{n}^{k}\right)-\sqrt{t_{n}^{k}-s_{n}^{k}} \cdot F_{n}(s)\right|<\sqrt{t_{n}^{k}-s_{n}^{k}} \cdot 2^{1-n} .
$$

Proof Using Lemma 3.6, we have $\left\|W(t)-F_{n}(t)\right\|_{\infty}<2^{-n}$ with positive probability, see Fig. 1.

Let $H_{n}^{k}:[0,1] \rightarrow A_{n}^{k}$ be the map defined by $x \mapsto\left|A_{n}^{k}\right| x+\inf A_{n}^{k}$. By the scaling property of Wiener processes, $W_{n}^{k}(s)=\left(W \circ H_{n}^{k}(s)-W \circ H_{n}^{k}(0)\right) / \sqrt{\left|A_{n}^{k}\right|}$ is equal in distribution to $W(s)$. Therefore, $\mathbb{P}\left\{\left\|W_{n}^{k}-F_{n}\right\|_{\infty}<2^{-n}\right\} \geq p_{n}>0$, where $p_{n}$ only depends on $n$. Further, the disjointness of intervals $A_{n}^{k}$ and $A_{n^{\prime}}^{k^{\prime}}$ for $(n, k) \neq\left(n^{\prime}, k^{\prime}\right)$ implies that the events are independent for different $(n, k)$. A simple application of the Borel-Cantelli lemma then shows that for every $n$ there are infinitely many $k$ such that $\left\|W_{n}^{k}-F_{n}\right\|_{\infty}<2^{-n}$ almost surely.

Let us define the Brownian bridge $B$ by $B(s)=W(s)-s W(1)$ and let

$$
B_{n}^{k}(s)=\left(B \circ H_{n}^{k}(s)-B \circ H_{n}^{k}(0)\right) / \sqrt{\left|A_{n}^{k}\right|}
$$




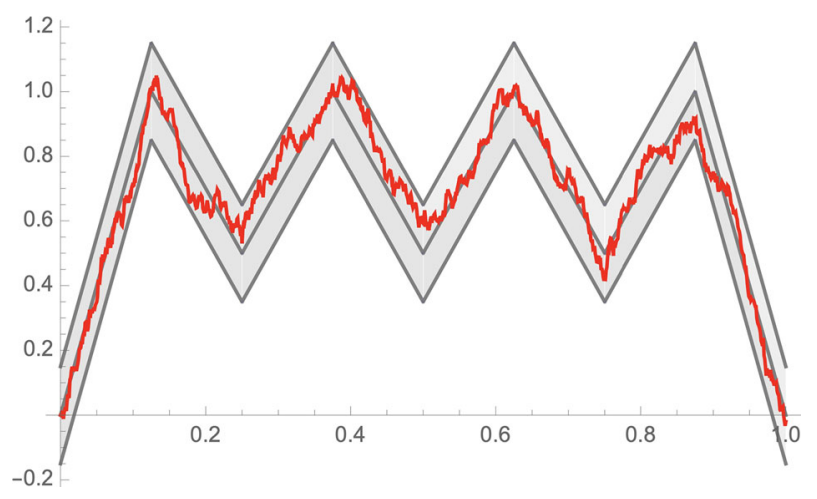

Fig. 1 The function $F_{4}$ and a Wiener process such that $\left\|W-F_{4}\right\|_{\infty}<1 / 8$

$$
=\left(W \circ H_{n}^{k}(s)-s W(1) \cdot H_{n}^{k}(s)-W \circ H_{n}^{k}(0)+s W(1) \cdot H_{n}^{k}(0)\right) / \sqrt{\left|A_{n}^{k}\right|} .
$$

While it is not true that $B_{n}^{k}(s)$ is independent of $B_{n^{\prime}}^{k^{\prime}}(t)$, the problematic $W(1)$ plays a diminishing role in approximating $B_{n}^{k}$ by $F_{n}^{k}$ for $n, k$ large.

Fix a realisation such that for all $n$ there are infinitely many $k$ such that $\left\|W_{n}^{k}-F_{n}\right\|<$ $2^{-n}$. Clearly this realisation is generic as the event has full measure. Then, for this realisation,

$$
\begin{aligned}
& \left\|B_{n}^{k}-F_{n}\right\|_{\infty} \\
= & \left\|\left(W \circ H_{n}^{k}-s \cdot W(1) \cdot H_{n}^{k}-W \circ H_{n}^{k}(0)+s \cdot W(1) \cdot H_{n}^{k}(0)\right) / \sqrt{\left|A_{n}^{k}\right|}-F_{n}\right\|_{\infty} \\
\leq & \left\|W_{n}^{k}-F_{n}\right\|_{\infty}+\left\|s \cdot W(1) \cdot\left(H_{n}^{k}(0)-H_{n}^{k}\right)\right\|_{\infty} / \sqrt{\left|A_{n}^{k}\right|} \\
\leq & 2^{-n}+\sqrt{\left|A_{n}^{k}\right|} \cdot|W(1)|=2^{-n}+2^{-(n+k) / 2-1}|W(1)| .
\end{aligned}
$$

Since $W(1)$ is fixed for this realisation we obtain that for every $n \in \mathbb{N}$ there are infinitely many $k \in \mathbb{N}$ such that $\left\|B_{n}^{k}-F_{n}\right\|<2^{-(n-1)}$. Finally, e is a simple cut and translate transformation of $B$, where the cut is the (almost surely) unique $t_{\min }$ with $B\left(t_{\min }\right)=\min _{t \in[0,1]} B(t)$. There are two cases to consider. Either $t_{\min } \geq 3 / 4$ or $t_{\min }<3 / 4$. In the former case we define the interval $I_{n}^{k}=A_{n}^{k}+1-t_{\min }$ and, in the latter case, we define $I_{n}^{k}=A_{n}^{k}-t_{\min }$ for $n$ large enough such that inf $A_{n}^{1}>t_{\min }$. Analogous to $H_{n}^{k}$, define $G_{n}^{k}:[0,1] \rightarrow I_{n}^{k}$ to be the unique orientation preserving similarity mapping $[0,1)$ into $I_{n}^{k}$. Therefore, almost surely, for large enough $n$ there exist infinitely many $k$ such that $\sup _{s \in[0,1)}\left|\left(\mathbf{e} \circ G_{n}^{k}(s)-\mathbf{e} \circ G_{n}^{k}(0)\right) / \sqrt{\left|I_{n}^{k}\right|}-F_{n}(s)\right|<$ $2^{1-n}$. This is the required conclusion.

Equipped with this lemma, we prove that the CRT is starry. 
Proof of Theorem 3.5 Let $G_{n}^{k}$ be as in the proof of Lemma 3.7 and assume that $\mathbf{e}$ is a generic realisation. Then,

$\mathbf{e} \circ G_{n}^{k}(0)+\sqrt{\left|I_{n}^{k}\right|} \cdot\left(F_{n}(s)-2^{1-n}\right) \leq \mathbf{e} \circ G_{n}^{k}(s) \leq \mathbf{e} \circ G_{n}^{k}(0)+\sqrt{\left|I_{n}^{k}\right|} \cdot\left(F_{n}(s)+2^{1-n}\right)$

for all $0 \leq s \leq 1$, where the existence of $k$ for large enough $n$ is guaranteed by Lemma 3.7. Let $z=1 /(4 n)$. Further, let $y_{p}=(2 p+1) / 2 n$ for $0 \leq p \leq n-2$. We now estimate the distances between $G_{n}^{k}(z)$ and $G_{n}^{k}\left(y_{p}\right)$ with respect to the $d_{\mathbf{e}}$ metric.

First, by 3.1,

$$
\begin{aligned}
& d_{\mathbf{e}}\left(G_{n}^{k}(z), G_{n}^{k}\left(y_{p}\right)\right) \\
& \quad=\mathbf{e}\left(G_{n}^{k}(z)\right)+\mathbf{e}\left(G_{n}^{k}\left(y_{p}\right)\right)-2 \min _{t \in\left[z, y_{p}\right]} \mathbf{e}\left(G_{n}^{k}(t)\right) \\
& \quad \leq \sqrt{\left|I_{n}^{k}\right|}\left(F_{n}(z)+F_{n}\left(y_{p}\right)+2 \cdot 2^{1-n}-2 \min _{t \in[z, 1-z]}\left(F_{n}(t)-2^{1-n}\right)\right) \\
& \quad=\sqrt{\left|I_{n}^{k}\right|}\left(2^{3-n}+1 / 2+1-2 \cdot 1 / 2\right)=\sqrt{\left|I_{n}^{k}\right|}\left(\frac{1}{2}+2^{3-n}\right)
\end{aligned}
$$

and

$$
\begin{aligned}
& d_{\mathbf{e}}\left(G_{n}^{k}(z), G_{n}^{k}\left(y_{p}\right)\right) \\
& \quad \geq \sqrt{\left|I_{n}^{k}\right|}\left(F_{n}(z)+F_{n}\left(y_{p}\right)-2 \cdot 2^{1-n}-2 \min _{t \in[z, 1-z]}\left(F_{n}(t)+2^{1-n}\right)\right) \\
& \quad=\sqrt{\left|I_{n}^{k}\right|}\left(-2^{3-n}+1 / 2+1-2 \cdot 1 / 2\right)=\sqrt{\left|I_{n}^{k}\right|}\left(\frac{1}{2}-2^{3-n}\right) .
\end{aligned}
$$

Similarly, for $q \neq p$,

$$
\begin{aligned}
& d_{\mathbf{e}}\left(G_{n}^{k}\left(y_{q}\right), G_{n}^{k}\left(y_{p}\right)\right) \\
& \quad=\mathbf{e}\left(G_{n}^{k}\left(y_{q}\right)\right)+\mathbf{e}\left(G_{n}^{k}\left(y_{p}\right)\right)-2 \min _{t \in\left[y_{q}, y_{p}\right]} \mathbf{e}\left(G_{n}^{k}(t)\right) \\
& \quad \leq \sqrt{\left|I_{n}^{k}\right|}\left(F_{n}\left(y_{q}\right)+F_{n}\left(y_{p}\right)+2 \cdot 2^{1-n}-2 \min _{t \in[z, 1-z]}\left(F_{n}(t)-2^{1-n}\right)\right) \\
& \quad=\sqrt{\left|I_{n}^{k}\right|}\left(2^{3-n}+1+1-2 \cdot 1 / 2\right)=\sqrt{\left|I_{n}^{k}\right|}\left(1+2^{3-n}\right)
\end{aligned}
$$

and

$$
d_{\mathbf{e}}\left(G_{n}^{k}\left(y_{q}\right), G_{n}^{k}\left(y_{p}\right)\right) \geq \sqrt{\left|I_{n}^{k}\right|}\left(1-2^{3-n}\right) .
$$

Let $\varrho_{n}=\left(1-2^{4-n}\right) \frac{1}{2} \sqrt{\left|I_{n}^{k}\right|}, A_{n}=\left(2+2^{4-n}\right) /\left(1-2^{4-n}\right)$, and $\eta_{n}=\left(1+2^{4-n}\right) /(1-$ $\left.2^{4-n}\right)-1$. For $n \geq 6$ we obtain $A_{n}>2,0<\eta_{n} \leq 2 / 3$, and

$$
A_{n}-\eta_{n}=\left(2-2^{4-n}\right) /\left(1-2^{4-n}\right)>\left(1+2^{4-n}\right) /\left(1-2^{4-n}\right)=1+\eta_{n} .
$$


Therefore the points $x_{0}=G_{n}^{k}(z), x_{p}=G_{n}^{k}\left(y_{p}\right)(1 \leq p \leq n)$ form an $\left(A_{n}, \eta\right)$ approximate $(n-1)$-star for sufficiently large $n$ and so, as $A_{n}>2>1$, we conclude that $T_{\mathbf{e}}$ is starry almost surely.

\section{The Brownian map}

As mentioned in the introduction, the Brownian map is a model of random geometry of the sphere that arises as the limit of many models of planar maps, e.g. uniform $p$-angulations $(p \geq 3)$ of $\mathbb{S}^{2}$, as the number of vertices is increased. We refer the reader to the surveys [18] and [22] for a detailed description of the Brownian map and its relation to other random geometries, such as Liouville Quantum Gravity.

In this section we define the Brownian map in terms of the Brownian continuum random tree and show that the Brownian map is almost surely starry.

\subsection{Definition of the Brownian map}

To obtain the Brownian map from the CRT, one defines a random pseudometric on the CRT, which in turn results in a (quotient) metric space that is the Brownian map. This metric is defined in terms of a Gaussian process defined on the CRT.

Let $Z(t)$ be a centered Gaussian process conditioned on e such that the covariance satisfies

$$
\mathbb{E}(Z(s) Z(t) \mid \mathbf{e})=\min \{\mathbf{e}(u): \min \{s, t\} \leq u \leq \max \{s, t\}\}
$$

Note that this completely determines the Gaussian process and that the second moment of distances is equal to the distance on the CRT, that is $\mathbb{E}\left((Z(s)-Z(t))^{2} \mid \mathbf{e}\right)=d_{\mathbf{e}}(s, t)$. One may imagine this process as a Wiener process along geodesics of the CRT, where branches evolve independently up to the common joint value.

We use this process to define a pseudo metric on $[0,1]$ by first setting

$$
D^{o}(s, t)=Z(s)+Z(t)-2 \max \left\{\min _{u \in[s, t]} Z(u), \min _{u \in[t, s]} Z(u)\right\}
$$

for $s, t \in[0,1]$. We can also define $D^{o}$ directly on the tree $T_{\mathrm{e}}$ by defining

$$
D^{o}(x, y)=\min \left\{D^{o}(s, t): \pi(s)=x \text { and } \pi(t)=y\right\}
$$

for $x, y \in T_{\mathbf{e}}$, where $\pi:[0,1] \rightarrow T_{\mathbf{e}}$ is the canonical projection under the equivalence relation $\approx_{\mathbf{e}}$, cf. Definition 3.3. Note that $D^{o}$ does not satisfy the triangle inequality and for $s, t \in[0,1]$ we further define

$$
D(s, t)=\inf \left\{\sum_{i=0}^{n-1} D^{o}\left(u_{i}, u_{i+1}\right): u_{i} \in T_{\mathbf{e}}\right\},
$$




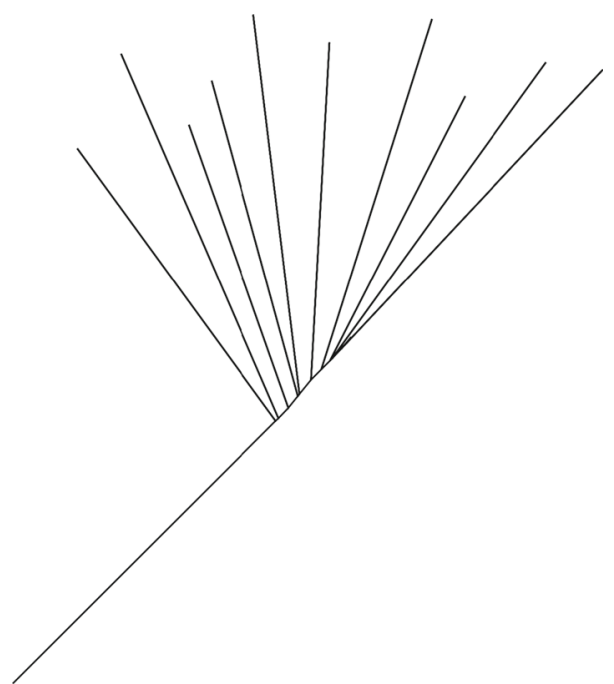

Fig. 2 The metric structure of an $F_{10}$ approximation

where the infimum is taken over all finite choices of $u_{i}$ satisfying $\pi(s)=u_{0}$ and $\pi(t)=u_{n}$. It can be verified that $D$ is indeed a pseudometric on $[0,1]$. Considering $\mathbf{m}_{\infty}=[0,1] / \approx$, where $s \approx t$ if and only if $D(s, t)=0$ we obtain the Brownian map $\left(\mathbf{m}_{\infty}, D\right)$. More generally, we can construct a Brownian map for any excursion function $f$ by replacing e with $f$ in the construction above. We call the resulting metric space $(\Phi(f), D)$ the Brownian map conditioned on the excursion function $f$. Letting $f=\mathbf{e}$, we recover the Brownian map $\left(\mathbf{m}_{\infty}, D\right)=(\Phi(\mathbf{e}), D)$.

\subsection{The Brownian map is starry}

Lemma 3.7 gives an insight in the extremal structure of $T_{\mathbf{e}}$ and we will see that it also implies extremal behaviour for the Brownian map. The approximate star for the CRT (see Fig. 2 gives rise to a line segment of length approximately $\varrho$ to which $n-1$ line segments of length approximately $\varrho$ are glued near the end.

By a similar argument to the one in Lemma 3.7 we will see that there exists positive probability that the Gaussian process defined on this substructure follows a similar "zig-zag" pattern that gives rise to a further approximate $(n-1)$-star, see Fig. 3.

Theorem 4.1 Let $f:[0,1] \rightarrow \mathbb{R}$ be an excursion function such that for infinitely many $n \in \mathbb{N}$, there exists infinitely many intervals $\left[s_{n}^{k}, t_{n}^{k}\right] \in[0,1], k \in \mathbb{N}$, such that

$$
\sup _{s \in\left[s_{n}^{k}, t_{n}^{k}\right]}\left|f(s)-f\left(s_{n}^{k}\right)-\sqrt{t_{n}^{k}-s_{n}^{k}} \cdot F_{n}\left(\left(s-s_{n}^{k}\right) /\left(t_{n}^{k}-s_{n}^{k}\right)\right)\right|<\sqrt{t_{n}^{k}-s_{n}^{k}} \cdot 2^{1-n}
$$

where the intervals $\left[s_{n}^{k}, t_{n}^{k}\right]$ are pairwise disjoint. Then, $(\Phi(f), D)$ is almost surely starry. 
In particular, this is true for almost every realisation of the Brownian excursion, see Lemma 3.7.

Corollary 4.2 The Brownian map $\left(\mathbf{m}_{\infty}, D\right)$ is almost surely starry and thus for all $0<\alpha \leq 1$, doubling metric spaces $(X, d)$ and increasing functions $\Psi:(0, \infty) \rightarrow$ $(0, \infty)$ any homeomorphism $\phi:\left(\mathbf{m}_{\infty}, D\right) \rightarrow(X, d)$ cannot satisfy

$$
\frac{d(\phi(x), \phi(y)}{d(\phi(x), \phi(z))} \leq \Psi\left(\frac{D(x, y)^{\alpha}}{D(x, z)^{\alpha}}\right)
$$

for all $x, y, z \in \mathbf{m}_{\infty}$.

Note further that the intervals at which we chose to "zoom in" on the CRT and Brownian map are arbitrary. We can easily make the stronger statement that there cannot be a single neighbourhood at which the homeomorphism can be quasisymmetric, i.e. the map cannot even be locally quasisymmetric.

\subsection{Proofs of Sect. 4}

Proof of Theorem 4.1 Temporarily fix $n, k$ such that (4.1) holds. Define

$$
\begin{aligned}
& x_{0}=x_{n}=\operatorname{argmax}\left\{\min \left\{f(s): 0 \leq s-s_{n}^{k} \leq\left(t_{n}^{k}-s_{n}^{k}\right) /(2 n)\right\}, \min \{f(s):\right. \\
& \left.\left.(1-1 /(2 n))\left(t_{n}^{k}-s_{n}^{k}\right) \leq s-s_{n}^{k} \leq\left(t_{n}^{k}-s_{n}^{k}\right)\right\}\right\}, \\
& x_{p}=\operatorname{argmin}\left\{f(s): \frac{p-1 / 2}{n}\left(t_{n}^{k}-s_{n}^{k}\right) \leq s-s_{n}^{k} \leq\right. \\
& \left.\frac{p+1 / 2}{n}\left(t_{n}^{k}-s_{n}^{k}\right)\right\} \text { for } 1 \leq p \leq n-1,
\end{aligned}
$$

and

$y_{p}=\operatorname{argmax}\left\{f(s): \frac{p}{n}\left(t_{n}^{k}-s_{n}^{k}\right) \leq s-s_{n}^{k} \leq \frac{p+1}{n}\left(t_{n}^{k}-s_{n}^{k}\right)\right\} \quad$ for $\quad 0 \leq p \leq n-1$.

Consider the "subtree" $\left(\left[s_{n}^{k}, t_{n}^{k}\right], d_{f}\right) \subset\left(T_{f}, d_{f}\right)$ and note that it contains $n+1$ lines $L_{i}$ of length comparable to $(1 / 2) \sqrt{t_{n}^{k}-s_{n}^{k}}$. They are

$$
L_{i}=\left\{\max \left\{s \in\left[x_{i}, y_{i}\right]: f(s)=t\right\}: t \in\left[\max \left\{f\left(x_{i}\right), f\left(x_{i+1}\right)\right\}, f\left(y_{i}\right)\right]\right\}
$$

and we have $f\left(L_{i}\right)=\left[\max \left\{f\left(x_{i}\right), f\left(x_{i+1}\right)\right\}, f\left(y_{i}\right)\right]$, sup $L_{i} \leq \inf L_{j}$ for $i<j$, $\min _{s \in L_{i}} f(s)=f\left(\inf L_{i}\right)$, and $\left.f\right|_{L_{i}}$ is a bijection. This structure can easily be seen on Fig. 3 (left). The point $x_{n}=x_{0}$ corresponds to the root of the tree, the $x_{i}$ correspond to the branch points of line segment $L_{i}$ that end at the leaf $y_{i}$.

The Gaussian $Z$ on $[0,1]$ has the property that $\left.Z\right|_{L_{i}}$ is independent of $\left.Z\right|_{L_{j}}$ if $i \neq j$ and is a Wiener process on $f\left(L_{i}\right)$. Let $s \in L_{i}, t \in L_{j}$ and assume without loss of generality that $s<t$. Then, 


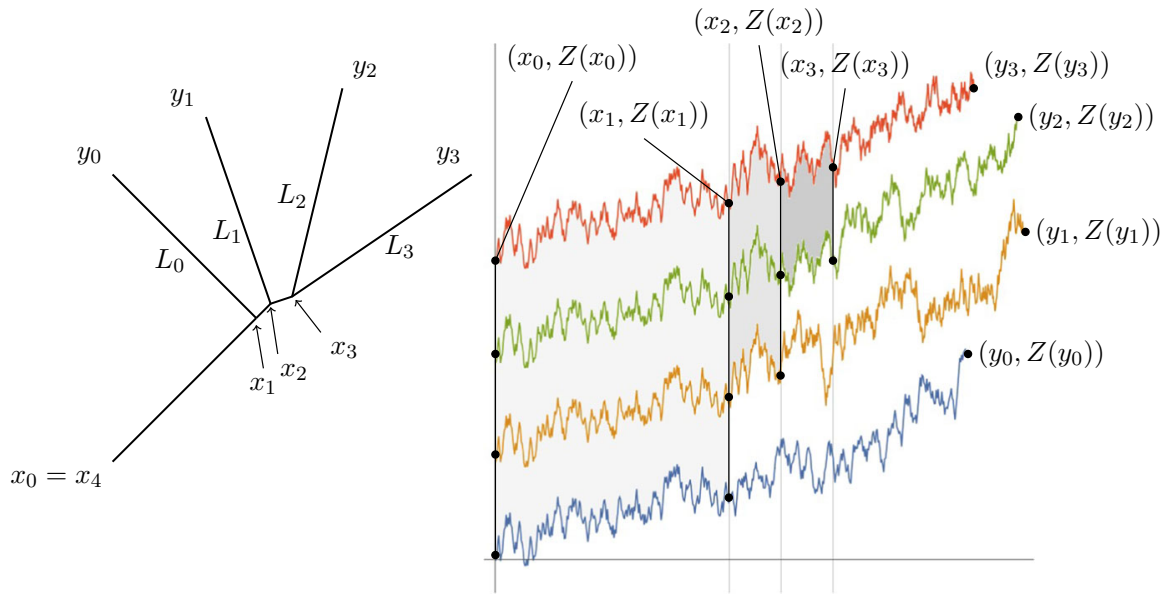

Fig. 3 Labelled structure of an $F_{4}$ approximation (left) with line segments $L_{0}, L_{1}, L_{2}$. Gaussian process indexed by the $F_{4}$ subtree (right). From top to bottom, the four graphs show the biased Gaussian process $Z$ along the geodesic from $x_{0}$ to $y_{3}, y_{2}, y_{1}$, and $y_{0}$, respectively

$$
\begin{aligned}
\operatorname{Cov} & \left(Z(s)-Z\left(\inf L_{i}\right), Z(t)-Z\left(\inf L_{j}\right)\right) \\
= & \operatorname{Cov}(Z(s), Z(t))-\operatorname{Cov}\left(Z(s), Z\left(\inf L_{j}\right)\right)-\operatorname{Cov}\left(Z(t), Z\left(\inf L_{i}\right)\right) \\
& +\operatorname{Cov}\left(Z\left(\inf L_{i}\right), Z\left(\inf L_{j}\right)\right) \\
= & \min _{u \in[s, t]} f(u)-\min _{u \in\left[s, \inf L_{j}\right]} f(u)-\min _{\left[\inf L_{i}, t\right]} f(u)+\min _{\left[\inf L_{i}, \inf L_{j}\right]} f(u) \\
= & \min _{u \in\left[s, \inf L_{j}\right]} f(u)-\min _{u \in\left[s, \inf L_{j}\right]} f(u) \\
& -\min _{u \in\left[\inf L_{i}, \inf L_{j}\right]} f(u)+\min _{u \in\left[\inf L_{i}, \inf L_{j}\right]} f(u)=0 .
\end{aligned}
$$

Now let $s, t \in L_{i}$. Then,

$$
\begin{aligned}
& \operatorname{Cov}(Z(s), Z(t))=\min \{f(u): \min \{s, t\} \leq u \leq \max \{s, t\}\} \\
& =f(\min \{s, t\})=\min \{f(s), f(t)\}
\end{aligned}
$$

Let $G_{i}$ be the unique orientation preserving similarity mapping the interval $[0,1]$ onto $f\left(L_{i}\right)=\left[\max \left\{f\left(x_{i}\right), f\left(x_{i+1}\right)\right\}, f\left(y_{i}\right)\right]$. Then,

$$
Z_{i}(s)=\left(Z \circ f^{-1} \circ G_{i}(s)-Z \circ f^{-1} \circ G_{i}(0)\right) / \sqrt{\left|f\left(L_{i}\right)\right|}
$$

is equal in distribution to a Wiener process $W$ and, as $\left|f\left(L_{i}\right)\right| \sim\left|f\left(L_{j}\right)\right|$, there exists $p_{n}$ independent of $i$ such that

$$
\mathbb{P}\left\{\left\|Z_{i}(s)-s\right\|_{\infty}<2^{-n}\right\} \geq p_{n}>0 .
$$

We can argue similarly for all the joined pieces $J_{i}$. They are independent (conditioned on starting value) and have length at most $\left(1+2^{1-n}\right)\left|f\left(L_{0}\right)\right|$. Hence the probability 
that the process deviates at most $2^{-n}$ is also comparable to $p_{n}$ and we assume without loss of generality that $p_{n}$ is a lower bound. Thus, the probability that this holds for all $i$ and all connecting tree pieces is at least $p_{n}^{n+1+\log _{2}(n+1)}>0$.

We now establish that these events are independent for distinct intervals. Let $x_{0}(n, k)$ be as in (4.2) and let $\left[s_{0}(n, k), t_{0}(n, k)\right] \subseteq\left[s_{n}^{k}, t_{n}^{k}\right]$ be the smallest interval such that $f\left(s_{0}(n, k)\right)=f\left(t_{0}(n, k)\right)=x_{0}$. Therefore $f(u) \geq x_{0}$ for all $u \in\left(s_{0}(n, k), t_{0}(n, k)\right)$. Assume that at least one of $n \neq n^{\prime}$ and $k \neq k^{\prime}$ is given, then the following holds for all $u_{1}, u_{2} \in\left[s_{0}(n, k), t_{0}(n, k)\right]$ and $v_{1}, v_{2} \in\left[s_{0}\left(n^{\prime}, k^{\prime}\right), t_{0}\left(n^{\prime}, k^{\prime}\right)\right]$, assuming without loss of generality that $t_{0}(n, k) \leq s_{0}\left(n^{\prime}, k^{\prime}\right)$,

$$
\begin{aligned}
\operatorname{Cov} & \left(Z\left(u_{1}\right)-Z\left(u_{2}\right), Z\left(v_{1}\right)-Z\left(v_{2}\right)\right) \\
= & \min _{u \in\left[u_{1}, v_{1}\right]} f(u)-\min _{u \in\left[u_{1}, v_{2}\right]} f(u)-\min _{u \in\left[u_{2}, v_{1}\right]} f(u)+\min _{u \in\left[u_{2}, v_{2}\right]} f(u) \\
= & \min _{u \in\left[t_{0}(n, k), s_{0}\left(n^{\prime}, k^{\prime}\right)\right]} f(u)-\min _{u \in\left[t_{0}(n, k), s_{0}\left(n^{\prime}, k^{\prime}\right)\right]} f(u) \\
& -\min _{u \in\left[t_{0}(n, k), s_{0}\left(n^{\prime}, k^{\prime}\right)\right]} f(u)+\min _{u \in\left[t_{0}(n, k), s_{0}\left(n^{\prime}, k^{\prime}\right)\right]} f(u) \\
= & 0 .
\end{aligned}
$$

Thus the (relative) Gaussian processes chosen earlier are independent if at least one of $n, n^{\prime}$ or $k, k^{\prime}$ differ. Thus a standard Borel-Cantelli argument shows that there are infinitely many intervals where this occurs for every $n$, almost surely.

The last thing to show is that these structures give rise to $(n-1)$-stars. This involves estimating the Brownian map metric as well as the following estimates. Let $x, y \in \mathbf{m}_{\infty}$, then

$$
Z(x)+Z(y)-2 Z(x \wedge y) \leq D(x, y) \leq D^{o}(x, y),
$$

where $x \wedge y$ is the lowest common ancestor of $x$ and $y$ in $T_{\mathbf{e}}$ and $\min _{z \in[x, y]} Z(z) \geq$ $\min _{z \in[0,1]} Z(z)$. We thus estimate, for $i \neq j$,

$$
\begin{aligned}
0 \leq D\left(x_{i}, x_{j}\right) & \leq D^{o}\left(x_{i}, x_{j}\right)=Z\left(x_{i}\right)+Z\left(x_{j}\right)-2 \min _{s \in\left[x_{i}, x_{j}\right]} Z(s) \\
& \leq 2 \max _{k} Z\left(x_{k}\right)-2 \min _{k} Z\left(x_{k}\right) \leq 4 \cdot 2^{-n} \max _{k} \sqrt{\left|f\left(L_{k}\right)\right|}
\end{aligned}
$$

as well as for the branches

$$
\begin{aligned}
D\left(x_{1}, y_{i}\right) & \leq D\left(x_{1}, x_{i}\right)+D\left(x_{i}, y_{i}\right) \\
& \leq 4 \cdot 2^{-n} \max _{k} \sqrt{\left|f\left(L_{k}\right)\right|}+\left(1+2 \cdot 2^{-n}\right) \sqrt{\left|f\left(L_{i}\right)\right|} \\
& \leq\left(1+6 \cdot 2^{-n}\right) \max _{k} \sqrt{\left|f\left(L_{k}\right)\right|}
\end{aligned}
$$

and 


$$
\begin{aligned}
D\left(y_{i}, y_{j}\right) & \leq D\left(x_{i}, x_{j}\right)+D\left(x_{i}, y_{i}\right)+D\left(x_{j}, y_{j}\right) \\
& \leq\left(2+16 \cdot 2^{-n}\right) \max _{k} \sqrt{\left|f\left(L_{k}\right)\right|} .
\end{aligned}
$$

The lower bounds are given by

$$
\begin{aligned}
D\left(x_{1}, y_{i}\right) & \geq Z\left(y_{i}\right)+Z\left(x_{1}\right)-2 Z\left(x_{1}\right)=Z\left(y_{i}\right)-Z\left(x_{1}\right) \\
& \geq Z\left(y_{i}\right)-Z\left(x_{i}\right)-\left|Z\left(x_{1}\right)-Z\left(x_{i}\right)\right| \\
& \geq \sqrt{\left|f\left(L_{i}\right)\right|}-2 \cdot 2^{-n} \max _{k} \sqrt{\left|f\left(L_{k}\right)\right|} \\
& \geq\left(1-6 \cdot 2^{-n}\right) \max _{k} \sqrt{\left|f\left(L_{k}\right)\right|}
\end{aligned}
$$

and

$$
D\left(y_{i}, y_{j}\right) \geq Z\left(y_{i}\right)+Z\left(y_{j}\right)-2 Z\left(y_{i} \wedge y_{j}\right) \geq\left(2-16 \cdot 2^{-n}\right) \max _{k} \sqrt{\left|f\left(L_{k}\right)\right|} .
$$

Analogous to the proof of Theorem 3.5 we see that the collection of points $y_{i}$ together with centre $x_{1}$, form an approximate $(n-1)$-star. Since this is true, almost surely, for every $n$ we have shown that $\left(\mathbf{m}_{\infty}, D\right)$ is starry.

\section{The continuum tree as a dual space}

While it is not generally true that the operator mapping continuous excursion functions on $[0,1]$ to continuum trees is invertible, one can see the tree associated with a function as an indicator of its irregularity. For instance, local maxima of the excursion function are exactly the leaves of the associated continuum tree (with the exception of the root). One might conjecture a strong link between dimensions of the graph of an excursion function and its associated continuum tree.

Question 5.1 Let $f$ be an excursion function on $[0,1]$ with graph $\mathcal{G}_{f}=\{(t, f(t))$ : $t \in[0,1]\} \subseteq \mathbb{R}^{2}$. Suppose additionally that $\operatorname{dim} \mathcal{G}_{f}=s$. What can we say about the dimension ${ }^{5} \operatorname{dim} T_{f}$ ?

Given the extremal nature of the Assouad dimension one might further conjecture that functions whose graph has Assouad dimension strictly larger than 1 have an associated continuum tree that is starry.

Conjecture 5.2 Let $f$ be an excursion function such that $\operatorname{dim}_{\mathrm{A}} \mathcal{G}_{f}>1$. Then $T_{f}$ is starry.

This is certainly not a necessary condition as Example 5.1 below shows.

Establishing such relationships between dimensions and irregular curves is at the heart of fractal geometry, see e.g. [7] and [9]. The dimension theory of the family of

\footnotetext{
${ }^{5}$ Here, dimension refers to any of the commonly considered dimensions: Hausdorff, packing, box-counting, Assouad, and lower dimension. 


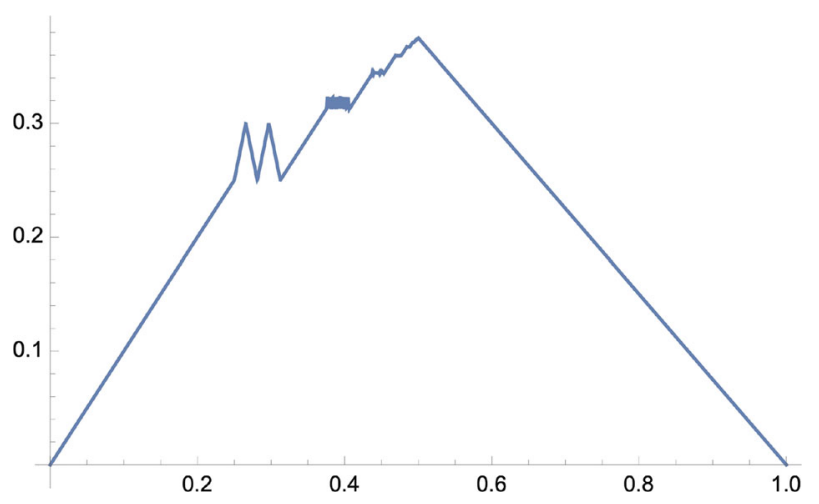

Fig. 4 The excursion function $g(x)+s(x)$

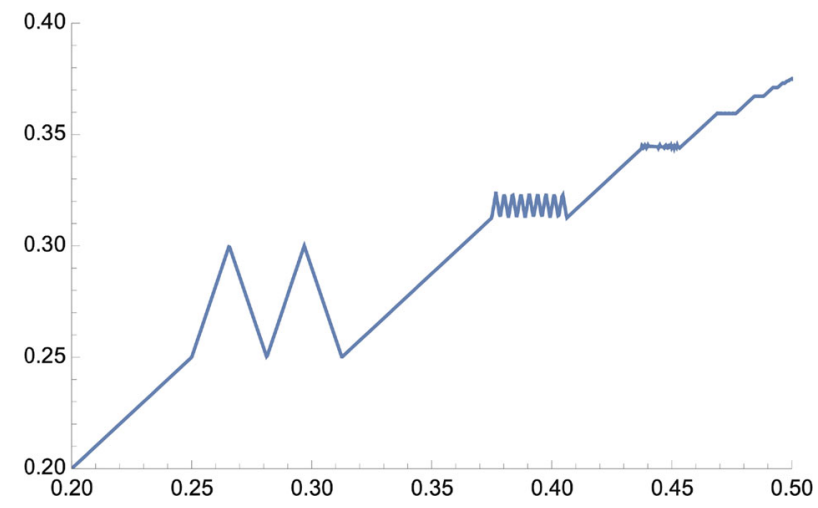

Fig. 5 Close up of Fig. 4

Weierstraß functions has only recently been analysed and self-affine curves are still not fully understood, see e.g. [6]. One of the major difficulty for affine functions are complicated relationships between the scaling in the horizontal as well as vertical axis and the continuum tree might get around this problem by separating the two scales in terms of placement of nodes and length of subtrees. These may give rise to a selfsimilar set in an abstract space other than $\mathbb{R}^{d}$ that may be easier to understand. Knowing bounds such as those asked in Question 5.1 may lead to a better understanding of the self-affine theory and this "dual space" could solve many open questions for singular functions.

\section{Example 5.1: Excursion with low Assouad dimension whose continuum tree is infinite dimensional}

Let $s_{n}(x)$ be the positive triangle function with slope 2 and $n$ maxima,

$$
s_{n}(x)= \begin{cases}2\left(x-\frac{k}{n}\right) & x \in\left[\frac{k}{n}, \frac{k}{n}+\frac{1}{2 n}\right) \quad \text { and } 0 \leq k<n \\ -2\left(x-\frac{k+1}{n}\right) & x \in\left[\frac{k}{n}+\frac{1}{2 n}, \frac{k+1}{n}\right) \quad \text { and } 0 \leq k<n \\ 0 & x=1\end{cases}
$$




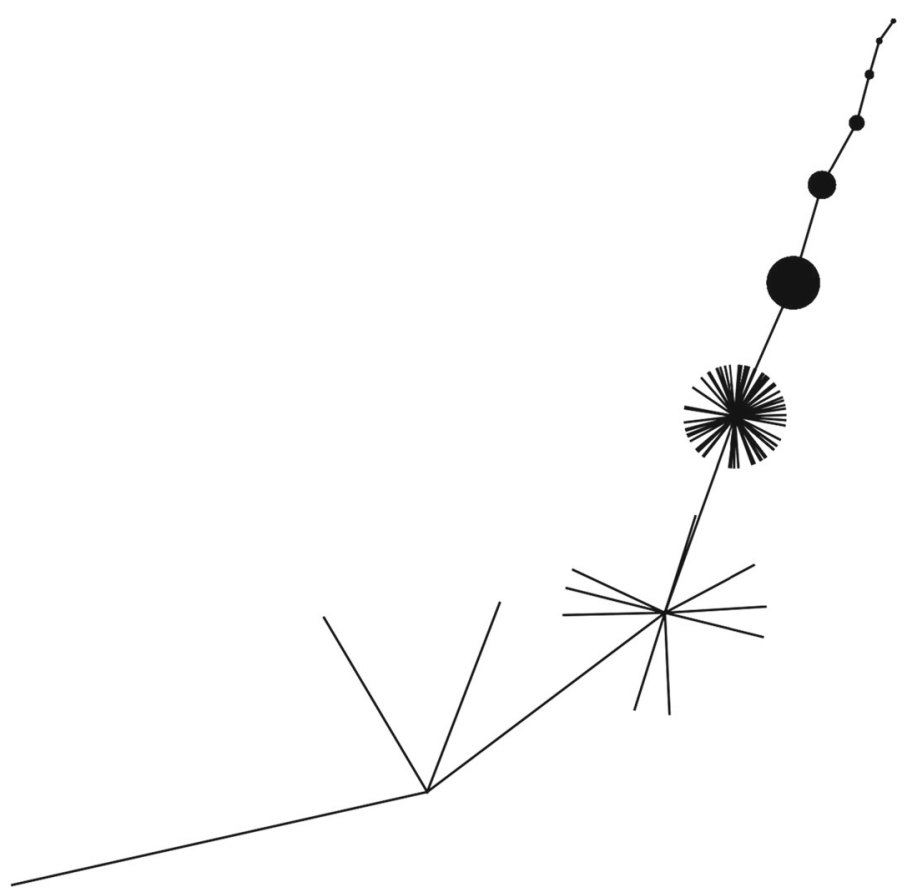

Fig. 6 The continuum tree $T_{g(x)+s(x)}$

Let us define disjoint dyadic intervals $\left[a_{n}, b_{n}\right] \subset[0,1 / 2]$ by $a_{n}=1 / 2-2^{-(n+1)}$ and $b_{n}=a_{n}+2^{-(n+3)}$. Note that $a_{n+1}-b_{n}=b_{n}-a_{n}$, and so, the gap between $\left[a_{n}, b_{n}\right]$ and $\left[a_{n+1}, b_{n+1}\right]$ is equal in size to the length of the former interval. We define

$$
s(x)=\sum \chi_{\left[a_{n}, b_{n}\right]}(x) \cdot\left(b_{n}-a_{n}\right) \cdot s_{(n+1)^{n}}\left(\left(x-a_{n}\right) /\left(b_{n}-a_{n}\right)\right)
$$

and note that this function is triangular on $\left[a_{n}, b_{n}\right]$ with slope 2 but decreasing amplitude and increasing frequency, such that it has $(n+1)^{n}$ maxima on $\left[a_{n}, b_{n}\right]$. The map $S:(x, y) \mapsto(x, y+s(x))$ is easily shown to be bi-Lipschitz on $[0,1] \times \mathbb{R}$ with Lipschitz constant $\sqrt{5}$. Further, let

$$
g_{1}(x)=\int_{0}^{x}\left(1-\sum_{n=1}^{\infty} \chi_{\left[a_{n}, b_{n}\right]}(y)\right) d y
$$

It is clear that $g_{1}(x)$ is a non-decreasing function with slope 0 in $\left[a_{n}, b_{n}\right]$ and slope 1 otherwise. Let $g_{2}(x)=-2 g_{1}(1 / 2) x+2 g_{1}(1 / 2)$ and

$$
g(x)= \begin{cases}g_{1}(x) & 0 \leq x<1 / 2 \\ g_{2}(x) & 1 / 2 \leq x \leq 1\end{cases}
$$


Similarly, $G:(x, y) \mapsto(x, y+g(x))$ is bi-Lipschitz on $[0,1] \times \mathbb{R}$ with Lipschitz constant $\sqrt{2}$. It can be checked that $g(x)+s(x)$ is an excursion function on $[0,1]$ see also Fig. 4 and Fig. 5. The graph $\{(x, g(x)+s(x)): x \in[0,1]\}$ is the image of $[0,1] \times\{0\}$ under $S \circ G$ and hence is a bi-Lipschitz image of the unit line. Therefore the Assouad dimension of the excursion graph $g(x)+s(x)$ is 1, i.e. as low as possible for a continuous function. To see that the resulting continuum tree $T_{g(x)+s(x)}$ is starry, one needs to observe that the triangle function gives the excursion functions $(n+1)^{n}$ peaks in $\left[a_{n}, b_{n}\right]$ whose amplitude is small compared to $g\left(a_{n+1}\right)$. This gives rise to approximate $(n+1)^{n}$-stars centred at $a_{n} \in T_{g(x)+s(x)}$ for all $n$ and thus $T_{g(x)+s(x)}$ is starry. See Fig. 6 for an illustration of $T_{g(x)+s(x)}$.

Acknowledgements The work on the BCRT first arose from a conversation with Noah Forman while the author was visiting the University of Washington in April 2018. ST thanks the University of Washington, and Jayadev Athreya and Noah Forman in particular, for the financial support, hospitality, and inspiring research atmosphere. The extension to the Brownian map was inspired by a question of Xiong Jin at the Thermodynamic Formalism, Ergodic Theory and Geometry Workshop at Warwick University in July 2019 and ST thanks Xiong Jin for many helpful conversations. Finally, I thank the referee for their helpful comments on an earlier version of this manuscript.

Funding Open Access funding provided by University of Vienna.

Open Access This article is licensed under a Creative Commons Attribution 4.0 International License, which permits use, sharing, adaptation, distribution and reproduction in any medium or format, as long as you give appropriate credit to the original author(s) and the source, provide a link to the Creative Commons licence, and indicate if changes were made. The images or other third party material in this article are included in the article's Creative Commons licence, unless indicated otherwise in a credit line to the material. If material is not included in the article's Creative Commons licence and your intended use is not permitted by statutory regulation or exceeds the permitted use, you will need to obtain permission directly from the copyright holder. To view a copy of this licence, visit http://creativecommons.org/licenses/by/4.0/.

\section{References}

1. Angelevska, J., Käenmäki, A., Troscheit, S.: Self-conformal sets with positive Hausdorff measure. Bull. Lond. Math. Soc. 52(1), 200-223 (2020)

2. Aldous, D.: The continuum random tree I. Ann. Probab. 19(1), 1-28 (1991)

3. Aldous, D.: The continuum random tree II. An overview, Stochastic analysis. London Mathematical Society. Lecture Note Se., 167, Cambridge University Press, 23-70 (1991)

4. Aldous, D.: The continuum random tree III. Ann. Probab. 21(1), 248-289 (1993)

5. Assouad, P.: Espaces métriques, plongements, facteurs, Thèse de doctorat d'État, Publ. Math. Orsay 223-7769, Univ. Paris XI, Orsay (1977)

6. Bárány, B., Kiss, G., Kolossváry, I.: Pointwise regularity of parametrized affine zipper fractal curves. Nonlinearity 31(5), 1705-1733 (2018)

7. Bishop, C.J., Peres, Y.: Fractals in Probability and Analysis. Cambridge University Press, Cambridge (2017)

8. David, G., Snipes, M.: A constructive proof of the Assouad embedding theorem with bounds on the dimension, hal-00751548 (2012)

9. Falconer, K.: Fractal Geometry, 3rd edn. Wiley, Chichester (2014)

10. Fraser, J.M.: Assouad dimension and fractal geometry. Cambridge University Press, Tracts in Mathematics Series, 222 (2020)

11. Fraser, J.M.: Interpolating between dimensions. Proceedings of Fractal Geometry and Stochastics VI, Birkhäuser, Progress in Probability (2019)

12. Fraser, J.M., Miao, J.-J., Troscheit, S.: The Assouad dimension of randomly generated fractals. Ergodic Theory Dyn. Syst. 38(3), 982-1011 (2018) 
13. Fraser, J.M., Troscheit, S.: The Assouad spectrum of random self-affine carpets. Ergodic Theory Dyn. Syst. (to appear) (2020). arXiv:1805.04643

14. Fraser, J.M., Yu, H.: Arithmetic patches, weak tangents, and dimension. Bull. Lond. Math. Soc. 50, 85-95 (2018)

15. Gwynne, E., Miller, J., Sheffield, S.: The Tutte embedding of the Poisson-Voronoi tesselation of the Brownian disk converges to $\sqrt{8 / 3}$-Liouville quantum gravity. Ann. Probab. (to appear). (2018). arXiv: $1809.02091 \mathrm{v} 3$

16. Käenmäki, A., Ojala, T., Rossi, E.: Rigidity of quasisymmetric mappings on self-affine carpets. Int. Math. Res. Not. IMRN 12, 3769-3799 (2018)

17. Le Gall, J.-F.: Uniqueness and universality of the Brownian map. Ann. Probab. 41(4), 2880-2960 (2013)

18. Le Gall, J.-F.: Random geometry on the sphere. Proc. Int. Congr. Math. Seoul 2014, 421-442 (2014)

19. Lin, P., Rohde, S.: Conformal weldings of dendrites, preprint (2019)

20. Mackay, J.M., Tyson, J.T.: Conformal Dimension: Theory and Application, University Lecture Series 54, AMS (2010)

21. Miermont, G.: The Brownian map is the scaling limit of uniform random plane quadrangulations. Acta Math. 210, 319-401 (2013)

22. Miller, J.: Liouville quantum gravity as a metric space and a scaling limit. In: Proceedings of the International Congress of Mathematicians

23. Miller, J., Sheffield, S.: Liouville quantum gravity and the Brownian map I: the QLE( $8 / 3,0)$ metric. Invent. math. 219, 75-152 (2020)

24. Miller, J., Sheffield, S.: Liouville quantum gravity and the Brownian map II: geodesics and continuity of the embedding, preprint. (2016) 119 pp. arXiv:1605.03563

25. Miller, J., Sheffield, S.: Liouville quantum gravity and the Brownian map III: the conformal structure is determined, Probab. Theory Related Fields (to appear). (2016) 32 pp. arXiv:1608.05391

26. Stroock, D.W.: Probability Theory - an analytic view, 2nd edn. Cambridge University Press, Cambridge (2010)

27. Troscheit, S.: The quasi-Assouad dimension of stochastically self-similar sets. Proc. R. Soc. Edinb. Sect. A 1-15 (2019)

28. Troscheit, S.: Assouad spectrum thresholds for some random constructions, Canad. Math. Bull., (2019), 1-20, https://doi.org/10.4153/S0008439519000547.

29. Tyson, J.T.: Lowering the Assouad dimension by quasisymmetric mappings. Illinois J. Math. 45(2), 641-656 (2001)

Publisher's Note Springer Nature remains neutral with regard to jurisdictional claims in published maps and institutional affiliations. 